\title{
High BRCA1 Gene Expression Increases the Risk of Early Onset Distant Metastasis in ER+ Breast Cancers
}

\author{
Hui-Ju Chang \\ National Yang-Ming University \\ Ueng-Cheng Yang ( $\nabla$ yang@ym.edu.tw ) \\ National Yang-Ming University \\ Mei-Yu Lai \\ Academia Sinica \\ Chen-Hsin Chen \\ Academia Sinica \\ Yang-Cheng Fann \\ National Institutes of Health
}

Research article

Keywords: BRCA1, early breast cancer, distant metastasis-free survival, ER+, tamoxifen, cure model

Posted Date: December 1st, 2020

DOI: https://doi.org/10.21203/rs.3.rs-116024/v1

License: (c) (1) This work is licensed under a Creative Commons Attribution 4.0 International License. Read Full License

Version of Record: A version of this preprint was published at Scientific Reports on January 7th, 2022. See the published version at https://doi.org/10.1038/s41598-021-03471-w. 
3 High $B R C A 1$ gene expression increases the risk of early onset

$13{ }^{1}$ Institute of Biomedical Informatics, National Yang-Ming University (No. 155, Sec. 2 ,

14 Li-Nong street, Taipei, Taiwan 11221, R.O.C.)

$15{ }^{2}$ Center for Systems and Synthetic Biology, National Yang-Ming University (No. 155,

16 Sec. 2, Li-Nong street, Taipei, Taiwan 11221, R.O.C.)

$17{ }^{3}$ Institute of Statistical Science, Academia Sinica, (128 Academia Road, Section 2,

18 Taipei, Taiwan 115201, R.O.C. )

194 Graduate Institute of Epidemiology and Prevention Medicine, National Taiwan

20 University, (17 Hsu-Chow Road, Taipei, Taiwan 100025, R.O.C. )

215 Division of Intramural Research, National Institute of Neurological Disorders and

22 Stroke, National Institutes of Health (35 Convent Dr, Bethesda, MD 20892 , USA)

23

$24{ }^{*}$ Corresponding author:

25 Ueng-Cheng Yang

26 yang@ym.edu.tw 


\section{Abstract}

28 Background: More than $30 \%$ of $\mathrm{ER}^{+}$breast cancer patients developed distant metastasis

29 after adjuvant tamoxifen therapy. It has been shown that decreased BRCA1 protein

30 function confers anti-estrogen "resistance." Since the functional status of BRCA1 protein

31 auto-regulates its own gene expression, $B R C A 1$ transcript level should possibly be used

32 to assess its active protein levels. Thus, it is interesting to explore the potential links

33 between $B R C A 1$ gene expression status and the prognosis of $\mathrm{ER}^{+}$breast tumors.

34 Methods: Early-staged $\mathrm{ER}^{+}$Breast cancer microarray samples were selected from the

35 NCBI Gene Expression Omnibus (GEO) database. The aggressiveness of these tumors

36 was evaluated based on molecular subtypes or patients' distant metastasis-free survival

37 (DMFS) time found in associated GEO datasets. In survival analysis, the optimal

38 threshold to define high and low transcript levels was assessed by the logistic-accelerated

39 failure time mixture regression model in a pooled data set, which simultaneously

40 envisages the lifetime risk of distant metastasis and the distribution of the DMFS time

41 from the surgery of a patient susceptible to distant metastasis. 
42 Results: On the basis of molecular subtyping, the relatively aggressive Luminal B tumor

43 cells expressed significantly more $B R C A 1$ transcripts than the less aggressive Luminal A

44 tumor cells. This observation was supported by DMFS time analysis. When the upper $10 \%$

45 of the high $B R C A 1$ expression patients was compared with the rest of the patients, the

46 result was quite drastic. In patients susceptible to distant metastasis, the median onset

47 time of distant metastasis for high BRCAl expression group (2.2 years) was about

48 one-fifth of the BRCA1 low expression group (10.5 years).

49 Conclusions: High BRCA1 transcript level does not increase the incidence of distant

50 metastasis, but increases the risk of early onset distant metastasis in $\mathrm{ER}^{+}$breast cancers.

51 As new therapeutic drugs have been developed for tumors with BRCA function loss, our

52 findings suggest that $B R C A 1$ gene expression test not only is useful for predicting

53 patients' outcome but also can be used in treatment decision.

54 Key words: $B R C A 1$, early breast cancer, distant metastasis-free survival, $\mathrm{ER}^{+}$, tamoxifen,

55 cure model 


\section{Introduction}

57 Breast cancer has been the most common and the leading cause of cancer deaths in

58 women worldwide [1,2]. Most breast cancers are sporadic and have no family history.

59 Based on their molecular characteristics [3], breast cancer can be classified into different

60 subtypes. These subtypes may help predicting patient outcome and serve as targets for

61 adjuvant therapies. According to the American Cancer Society, two out of three breast

62 cancers are positive for hormone receptors and most of them are estrogen receptor

63 positive $\left(\mathrm{ER}^{+}\right)$. Binding to ER, estrogen may activate estrogen-ER signaling pathway and

64 promote breast tumor formation [4,5] or induce the proliferation of $\mathrm{ER}^{+}$breast tumor

65 cells [6-8]. Therefore, a standard adjuvant therapy to treat $\mathrm{ER}^{+}$breast cancers is to

66 suppress the estrogen-induced proliferation by anti-estrogen therapies, such as using

67 tamoxifen. However, despite the clinical success of tamoxifen, about $22-52 \%$ of early

$68 \mathrm{ER}^{+}$breast tumors exhibit recurrence in long-term follow up studies after this

69 anti-estrogen treatment [9]. What causes this resistance is not clear yet, but decreased

70 Breast cancer type 1 susceptibility protein (BRCA1) has been reported to confer 
71 tamoxifen resistance [10].

72 In addition to its well-known role in DNA damage repair [11], BRCA1 has many

73 important biological functions. One of them is to regulate mammary cell growth and

74 differentiation through interacting with estrogen-ER signaling pathway [12,13]. By

75 forming a complex with ER, BRCA1 induces ER conformation change and regulates

76 ER's transcriptional activity [12]. Loss of BRCA1 function increases ER-coactivator

77 association in tamoxifen treated cancer cells, which leads to tamoxifen resistance [10]. In

78 fact, decreased BRCA1 protein staining is commonly observed in sporadic breast cancers

$79[14,15]$ and this phenomenon is associated with high histologic grades [16-18] and short

80 disease-free survival [19]. It is certain that such association is related to an E-ER

81 signaling pathway.

82 If BRCA1 function does play a decisive role in breast cancer prognosis, this protein

83 should be a good predictor for patients' prognosis or even can serve as a target for

84 treatment. However, there are still many problems to be solved before this idea can be

85 applied clinically. One of them is how to develop a precise measuring tool to check the 
86 active BRCA1 protein level in a tumor. Gene-expression based methods, such as PCR,

87 could be good candidates, readily quantifiable, reliable, and cost-effective.

88 When the BRCA1 protein loses its function, a cell may undergo more mutations due to

89 defective DNA repair and be transformed into a cancer cell. As BRCA1 protein

90 auto-regulates its own gene transcription [20], this loss-of-function mutation may lead to

91 a high $B R C A 1$ gene expression. Therefore, high $B R C A 1$ expression might be associated

92 with aggressive behavior in breast tumors. This assumption was supported by previous

93 study results showing that BRCA1 mRNA level was up-regulated in tumors [21-23] and

94 that high BRCA1 gene expression was associated with fast metastasis [24]. However,

95 some earlier studies presented opposite findings [25-28]. These controversial

96 observations need to be resolved before exploring the possibility of using a

97 gene-expression test to check active BRCA1 protein function.

98 This study used gene expression microarrays from the public domain to explore the

99 association between BRCAl mRNA level and the aggressiveness of breast tumors.

100 Molecular subtypes and distant metastasis-free survival (DMFS) were used to assess the 
101 aggressiveness of tumors, respectively. Among all different molecular subtypes of breast

102 cancers, only luminal A and B subtypes were selected to compare BRCA1 mRNA levels

103 because both subtypes are $\mathrm{ER}^{+}$. In survival analysis, only early-stage $\mathrm{ER}^{+}$patients who

104 received standard tamoxifen treatment after surgery were included in the DMFS analysis

105 in attempt to attain homogeneity among study subjects. A "cure model" [29] was utilized

106 in this study to tackle the non-susceptibility of distant metastasis, which would be ignored

107 by the commonly-used Cox proportional hazards regression model [30] in survival 108 analysis.

\section{Materials and Methods}

\section{Study samples}

111 Microarray data used in this study were all from the Gene Expression Omnibus (GEO)

112 repository [31] located at the National Center for Biotechnology Information (NCBI). To

113 examine $B R C A 1$ gene expressions in molecular subtypes of $\mathrm{ER}^{+}$breast tumors, we chose

114 dataset GSE45827 [32], which is composed of 130 primary invasive breast cancers (41

115 Triple Negative, 30 Her2, 29 Luminal A and 30 Luminal B tumors) as well as 11 normal 
116 tissues and 14 cell lines using Affymetrix HG U133 plus 2.0 as the study platform.

117 Because this study focused on $\mathrm{ER}^{+}$tumors, only the BRCA1 expression levels of normal

118 breast tissue, Luminal A, and Luminal B tumors from GSE45827 were compared.

119 Two criteria were considered in selecting the datasets for survival analysis: the first was

120 to acquire datasets that tracked the survival time with related clinical data, and the second

121 was to use datasets with an identical technology platform for microarray gene expression

122 data. Ten breast cancer datasets with survival records, which used the U133A platform for

123 expression analysis, were thus selected from the GEO database (see clinical information

124 in Supplementary Table S1). However, because DMFS could be more directly associated

125 with tumor behavior, the DMFS time was used as the endpoint of patients' prognosis so

126 that only those datasets with the DMFS time were selected for our study. To make our

127 study samples homogenous, the following inclusion criteria were further used to select

$128 \mathrm{ER}^{+}$patients for early detection and treatment: 1) the tumor was localized at its original

129 locus, 2) the lymph nodes were free of tumor, and 3) the tumor size was not larger than 5

$130 \mathrm{~cm}$. Study subjects must have an estrogen receptor status and adjuvant treatment 
131 information. After thoroughly examining patients' clinical information, only 359 patients

132 in three GEO datasets (GSE12093 [33], GSE17705 [34] and GSE45255 [35]) were

133 entered into our survival analysis (see Supplementary Table S2).

\section{Microarray data analysis}

136 The microarray data were normalized by using the Bioconductor R "affy" package [36].

137 In order to minimize the false-negative and false-positive rates, the parameters were set

138 up based on Choe's [37] study. Namely, bgcorrect.method = "mas", pmcorrect.method

139 = "pmonly", normalize.method = "quantiles", and summary.method = "medianpolish"

140 were set up and input into the "affy" package's "expresso" function. All samples of

141 GSE45827 (on HG-U133 plus 2.0 platform) were normalized. The 573 samples pooled

142 from three chosen GEO datasets, GSE12093, GSE17705 and GSE45255 (on HG-U133A

143 platform), were normalized together. After the normalization, eligible study samples

144 satisfying the aforementioned inclusion criteria were selected for further analysis.

145 The R package "jetset" [38] was used to select the most representative probe set from 
146 multiple probe sets of a gene in the Affymetrix microarray. This program computes

147 "jetscores" and uses the probe set of the highest score for a gene designated for further

148 analysis. In Affymetrix HG U133 series, the $B R C A 1$ gene has two corresponding probe

149 sets, 204531_s_at and 211851_x_at with "jetscores" of 0.278 and 0.029 , respectively.

150 Therefore, 204531_s_at was substituted for BRCA1 gene expression in our analysis.

\section{Statistical methods}

\section{The Kruskal-Wallis test}

154 To compare the differences in the BRCA1 gene expression levels among several groups,

155 the nonparametric Kruskal-Wallis test was used to test the null hypothesis that these

156 independent samples came from the same population distribution.

\section{Logistic-accelerated failure time (AFT) mixture regression model}

159 We employed the logistic-AFT mixture regression model [29] to compare two 
160 Kaplan-Meier curves [39] representing the DMFS time between high-level and low-level

161 BRCA1 expressions, which was defined by a specified cut-off point of BRCA1

162 expression values, with only right censored data. A patient was either an event case with

163 distant metastasis or right censored with a follow-up DMFS time. Fitting a patient's

164 indicator covariate representing the high- $B R C A l$ expression into the mixture model

165 simultaneously estimates her lifetime risk probability of distant metastasis and the

166 distribution of her DMFS time if she would be susceptible to distant metastasis. A brief

167 introduction to the mixture model is as follows:

168 Let $T$ be the duration in years from the surgery to distant metastasis in breast cancer, and

169 the survival distribution at $t$ years $\operatorname{Pr}(T>t)$ indicates the probability that a patient's cancer

170 will not metastasized for $t$ years. The patient's eventual susceptibility to distant metastasis

171 is represented by the binary indicator $D, D=1$ indicating the patient is susceptible, $\mathrm{D}=0$

172 indicating the patient is not susceptible. The probability that a patient with a risk factor $Z$,

173 such as an indicator of high-BRCA1 expression, will not metastasized after $t$ years is

174 expressed as: 
176 where, for a given $Z, \operatorname{Pr}(D=0 \mid Z)$ is the lifetime DMFS probability and $\operatorname{Pr}(T>t \mid D=1, Z)$

177 is the conditional survival function at $t$ years for a susceptible patient.

178 The logistic-AFT mixture regression model [29] contains both a logistic regression

179 submodel

180

$$
\ln \left(\frac{\operatorname{Pr}(D=1 \mid Z)}{\operatorname{Pr}(D=0 \mid Z)}\right)=\beta^{\prime} Z,
$$

181 and an AFT location-scale regression submodel

$$
\ln T(Z)=\gamma^{\prime} Z+\exp \left(\alpha^{\prime} Z\right) \varepsilon .
$$

183 Parameters $\beta, \gamma$, and $\alpha$ are in the logistic, location, and scale regression parts,

184 respectively, and can be simultaneously estimated using the R package

185 "MixtureRegLTIC"[40]. For a specific value of $Z$, the logistic submodel provides a

186 parametric estimator for the lifetime DMFS probability $\operatorname{Pr}(D=0 \mid Z)$, which can also be

187 estimated nonparametrically by the tail of the Kaplan-Meier overall DMFS curve. In

188 contrast, the AFT submodel formulates the parametric distribution of the DMFS time in

189 the logarithm scale for a susceptible patient, while the Kaplan-Meier conditional DMFS

190 curve is displayed as its nonparametric counterpart. 
191 Since the log-logistic distribution was much better fitted to our pooled GSE breast cancer

192 datasets for the AFT submodel than the other generalized gamma distribution available in

193 the MixtureRegLTIC, we assumed that $T$ of a susceptible patient follows a log-logistic

194 distribution in the AFT location-scale regression submodel; that is, $\varepsilon$ is a logistic error

195 term with the density $f(\varepsilon)=e^{\varepsilon} /\left(1+e^{\varepsilon}\right)^{2}$.

196 According to the minimum Akaike information criterion (AIC), we determined the best

197 model selection of the covariate to be included in the suitable regression parts of the

198 logistic-AFT location-scale mixture regression model. To check the adequacy of the fitted

199 model for high and low BRCA1 expressions, we plotted the overall survival curves $\operatorname{Pr}(T>$

$200 t \mid Z)$ and the conditional curves $\operatorname{Pr}(T>t \mid D=1, Z)$ by superimposing on the corresponding

201 Kaplan-Meier overall and conditional curves to visualize the goodness-of-fit for the

202 best-fitted mixture model. The resultant p-value of the likelihood ratio test in the

203 best-fitted mixture model is used for evaluating the significance of the difference between

204 the Kaplan-Meier overall DMFS curves. 


\section{Results}

\section{High $B R C A 1$ gene expression is associated with aggressive behavior in}

\section{$209 \mathbf{E R}^{+}$breast tumors}

210 BRCA1 is known to regulate mammary cell proliferation through interacting with ER.

211 Thus, we focused on $\mathrm{ER}^{+}$tumors (molecular subtypes luminal A and B) to make our

212 samples homogeneous. The GEO dataset GSE45827 contains Affymetrix HG-U133 plus

2132.0 microarrays of normal breast tissues and primary breast tumors with different

214 molecular subtypes. It allows us to compare the BRCA1 expression levels in different

215 molecular subtypes of $\mathrm{ER}^{+}$tumors. In Figure 1, BRCA1 expression levels in luminal B

216 tumors were significantly higher than those in normal breast tissues or luminal A tumors.

217 In contrast, $B R C A 1$ expression levels were not different in normal tissue and luminal A

218 tumors. Luminal A tumors are defined as tumors which are positive for ER but low in

219 either HER2 or proliferation marker Ki-67 levels. These tumors grow slowly and have a

220 good prognosis. Luminal B tumors are also $\mathrm{ER}^{+}$but with a high level of proliferation 
221 marker Ki-67. In other words, luminal B tumors grow faster than luminal A tumors. Our

222 observation reveals that high $B R C A 1$ expression is associated with the proliferation of

$223 \mathrm{ER}^{+}$cells.

226 Figure 1. Boxplots of BRCA1 expression in normal breast tissue, Luminal A and

227 Luminal B tumors of GSE45827. Normal breast tissues (red), luminal A tumors (green)

228 and luminal B tumors (blue) are displayed from left to right. Each dot represents the

229 BRCA1 expression level of a certain sample. Pairwise p-values between different subtypes

230 were calculated using the Kruskal-Wallis test.

233 High $B R C A 1$ gene expression is associated with fast distant metastasis in

$234 \mathbf{E R}^{+}$early-stage breast cancers

235 Molecular subtyping clearly demonstrated the association between BRCA1 gene 
236 expression level and tumor aggressiveness. It is expected that this association can be

237 reflected in the patients' survival pattern. Thus, a pooled dataset with both microarray

238 data and the survival data were collected from the GEO repository. Since a patient may

239 not have a long-term survival once she develops a distant metastasis, the DMFS is a good

240 endpoint for the aggressiveness of tumors.

241 The BRCAl expression level is a continuous variable and the histogram, which is

242 composed of the BRCA1 expression levels of the pooled samples from the three GEO

243 datasets, is unimodal. To compare the differences in the DMFS time of high- or

244 low-BRCA1 expression groups, a threshold for $B R C A 1$ expression is needed. Different

245 deciles in the histogram of $B R C A l$ expression distribution were used to categorize the

246 patients into high- or low-expression groups, respectively.

247 Results from fitting the logistic-AFT mixture model were not significant for each cut-off

248 point ranging from the $1^{\text {st }}$ to $4^{\text {th }}$ deciles (results not shown). Results falling into the $5^{\text {th }}$ to

$2498^{\text {th }}$ deciles were reported in Figure 2A-D and Table 1, and those for the cutoff-point at the

$2509^{\text {th }}$ decile were displayed in Figure 3 and Table 2. In each of these figures, the left panel 
251 indicates the overall DMFS curves and the right panel the conditional DMFS curves. The

252 former displayed the DMFS pattern for all patients, and the latter showed the DMFS

253 distribution of the patients who would be susceptible to distant metastasis. The time-axis

254 of the conditional DMFS curve was plotted in a logarithmic scale and could be used to

255 estimate the median onset time of distant metastasis for susceptible patients.

256 The difference between the Kaplan-Meier overall DMFS curves (step functions) for these

257 two expression groups defined by a specified decile was then examined. It was noted that

258 divided by higher deciles (such as 70\% to 90\%), the corresponding DMFS curves of the

259 high-BRCA1 expression groups (the red step functions in the left panels of Figures $2 \mathrm{C}$,

$2602 \mathrm{D}$ and 3) flatten off, respectively. In the high $B R C A 1$ expression group, some patients

261 developed distant metastasis early on around the first seven years after surgery. In contrast,

262 the rest (more than 60\%) of the patients rarely metastasized in the 15-year follow-up. These

263 results implied that the hazard ratio of the high-expression group to the low one changed

264 over the follow-up course, so using the logistic-AFT mixture regression model [29] to fit

265 the pooled GEO data with $B R A C 1$, instead of the Cox proportional hazards model [30], 
266 was quite adequate.

267 The mixture regression model enabled us to predict the DMFS accurately so that the

268 resultant overall and conditional DMFS (smooth) curves defined at higher deciles derived

269 from the model fitting were much closer to the corresponding empirical Kaplan-Meier

270 overall and conditional DMFS curves (step functions). In addition to the significant

271 p-values resulting from the likelihood ratio test (LRT) for the mixture models (cut-off from

$27250 \%$ to $90 \%$ ), a cutoff at 60 to $90 \%$ (Tables 1 and 2 ) seems to emerge as a trend. The

273 Kaplan-Meier conditional DMFS curves for patients susceptible to distant metastasis (right

274 panels in Figure 2A to 2D and in Figure 3) found in the high- and low- BRCA1 expression

275 groups were also separated more distinctly with increasing cut-off values. Our model

276 fitting obviously confirmed the $B R C A 1$-gene expression dependency with increasing

$277 B R C A 1$-expression levels. This trend was revealed with the statistical significance of the

278 high-BRCA1 expression in the "Location Regression Part" in Tables 1 and 2, together

279 with the well-separated low- and high-BRCA1 expression (smooth) curves in Figures 2

280 and 3 . The separation due to a negative estimate of the location parameter resulted in a 
281 smaller median onset time of distant metastasis in the high- $B R C A 1$ expression group than

282 in the lower one. On the other hand, each of the "Scale Regression Part" in Tables 1 and 2

283 did not include the high-BRCA1 expression, so the shapes of the low- and high-BRCA1

284 expression conditional DMFS curves were similar.

285

286

287

Table 1. Analysis of the log-logistic-AFT mixture regression model stratified by BRCA1-level with different cut-off points.

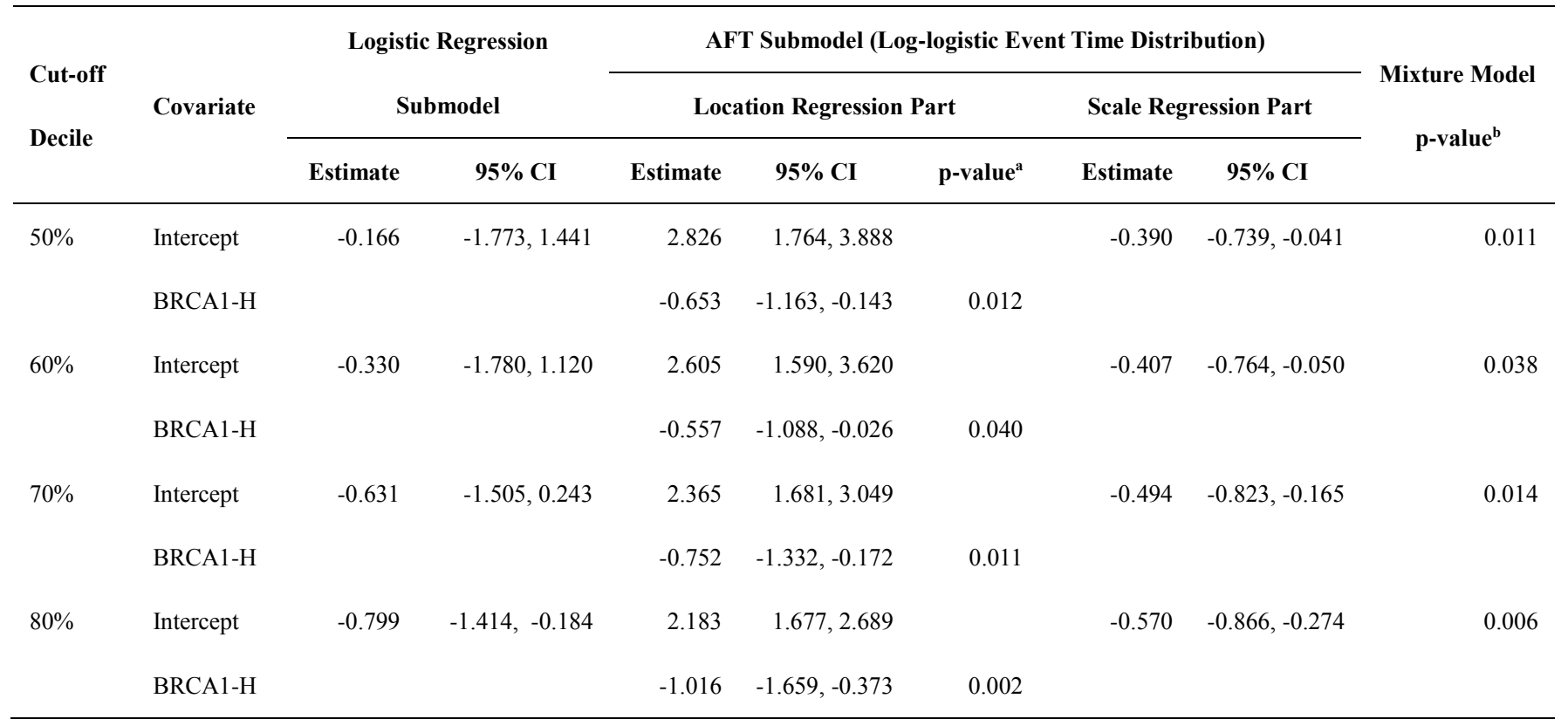


Abbreviations: CI, confidence interval; BRCA1-H, high BRCA1; LRT, likelihood ratio test.

p-value of Wald's statistic for testing the location parameter corresponding to BRCA1-H.

$\mathrm{p}$-value ${ }^{\mathrm{b}}$ of the LRT for testing the significance of the fitted mixture model.

290 Figure 2. Survival curves fitted by the "Logistic-AFT mixture regression model" at

291 the $\mathbf{5}^{\text {th }} \mathbf{8}^{\text {th }}$ deciles. Kaplan-Meier (step function) and the mixture regression (smooth

292 curve) estimators of overall and conditional DMFS curves for the distant metastasis onset

293 time after the surgery stratified by BRCA1-level defined at the cut-off point (A) $50^{\text {th }}$, (B)

$29460^{\text {th }},(\mathrm{C}) 70^{\text {th }}$, and (D) $80^{\text {th }}$ percentile, and corresponding $\mathrm{p}$ values of likelihood ratio test

295 ( $\left.\mathrm{P}_{\mathrm{LRT}}\right)$ were $0.011,0.038,0.014$ and 0.006 , respectively. The BRCA1 high- and

296 low-expression groups were shown in red and in blue, respectively, for each cut-off point.

299 Consequently, in Table 2 , the p value of the LRT corresponding to the $9^{\text {th }}$ decile was $<0.001$

300 (still significant even after adjustment for multiple comparison). It was the smallest among 
301 the LRT p-values for all deciles; it suggested that the best-fitted model was based on the

$30290^{\text {th }}$ percentile (of expression level 7.04) as the cut-off in the histogram for the pooled

$303 B R A C 1$ gene expression values. As shown in Figure 3, this cut-off point sharply contrasted

304 the difference for the two Kaplan-Meier DMFS curves depicting the high and low levels of

$305 B R C A 1$ expression. The decreasing trend of the AIC values (results not shown) among

306 increasing cut-off deciles implied that the possibility of over-fitting the model by using this

307 optimal cut-off point rather than other points was low.

308 The logistic regression submodel (Table 2) did not include the high-BRCA1 expression

309 variable. The estimated intercept (-0.520) indicated that the estimated common lifetime

310 probability of the DMFS in both expression levels was 0.627 . That is, $62.7 \%$ of the

311 early-stage $\mathrm{ER}^{+}$tamoxifen-treated breast cancer patients were free of distant metastasis

312 during the 15 years of follow-ups, no matter what the BRCA1 expression level was. It also

313 readily explained why the Kaplan-Meier overall DMFS curves depicting the $B R C A 1$ high-

314 (red step function) and low- (blue step function) expression groups in the left panel of

315 Figure 3 crossed at similar values of the DMFS proportion right before the $15^{\text {th }}$ year of 
316 follow-up. The location parameter in the AFT location-scale regression submodel,

317 however, included a high-BRCA1 expression variable with a negative parameter estimate

$318-1.571(\mathrm{p}<0.001$ of the Wald test, Table 2). This indicated that BRCA1 had an impact on

319 the distant metastasis after surgery time such that median onset time for susceptible

320 patients in the high-BRCAl expression group was significantly earlier than that of the low

321 expression group. The left panel of Figure 3 revealed that the Kaplan-Meier curve

322 illustrating the high $B R C A 1$ expression group (red step function) dropped sharply and then

323 flattened off afterwards. This was because distant metastasis in this group all occurred in

324 the first 7 years. Therefore, the rest of the patients appeared to be "cured" towards the end

325 of the 15-year follow-up period. Nevertheless, this phenomenon was not observed in the

326 low BRCA1 expression group because patients with low BRCAI expression gradually

327 developed distant metastasis, i.e., their relapses occurred in a steady rate during the 15

328 years. The median onset time of distant metastasis in the high- and low-expression groups

329 was estimated as 2.2 and 10.5 years, respectively. Because the median onset time of distant

330 metastasis for susceptible patients in the high $B R C A 1$ expression group was about one-fifth 
331 (2.2/ 10.5 years) of that in the low expression group, these patients seemed to have

332 extremely aggressive tumors. Therefore, $B R C A 1$ expression level may assist in identifying

333 these high-risk patients. Patients in the high $B R C A 1$ expression group should be treated

334 differently from other patients.

335

336

337 Figure 3. Survival curves fitted by the "Logistic-AFT mixture regression model" at

338 the $9^{\text {th }}$ decile. Kaplan-Meier (step function) and the mixture regression (smooth curve)

339 estimators of overall and conditional DMFS curves for the distant metastasis onset time

340 after the surgery stratified by BRCA1-level defined at the cut-off point 90th percentile.

341 The BRCAl high- and low-expression groups were shown in red and in blue, respectively. 
Table 2. Analysis of the logistic-AFT mixture regression model stratified by BRCA1-level cut-off at the 90th percentile.

\begin{tabular}{llrrr}
\hline \multirow{2}{*}{ Event Probability } & Covariates & Estimate & $\mathbf{9 5 \%}$ CI & p-value \\
\cline { 2 - 5 } Location & Intercept & -0.520 & $-1.218,0.178$ & 0.144 \\
& Intercept & 2.351 & $1.836,2.866$ & $<0.001$ \\
Scale & BRCA1-H & -1.571 & $-2.263,-0.879$ & $<0.001$ \\
\hline LRT mixture model & Intercept & -0.567 & $-0.841,-0.293$ & $<0.001$ \\
\hline
\end{tabular}

344 Abbreviations: CI, confidence interval; LRT, likelihood ratio test; BRCA1-H, high 345 BRCA1.

346

347 Discussion

348 Tamoxifen is usually used to treat the $\mathrm{ER}^{+}$patients. This drug is a selective estrogen

349 receptor modulator (SERM), competes with estrogen for ER-binding and inhibits the

350 proliferation stimulated by estrogen. It was reported that decreased BRCA1 expression

351 reduced the cellular response to tamoxifen [10] through increased ER-coactivator

352 association. As BRCA1 protein can auto-regulate its own gene transcription [20], a loss

353 of BRCA1 protein function may activate gene transcription and lead to high-BRCA1 gene

354 levels.

355 In previous studies of the association between $B R C A 1$ gene expression level and tumor 
356 behavior, conflicting observations came to light. Our results show that high BRCA1 gene

357 expression is associated with aggressive tumor behaviors and with fast distant metastasis

358 in early stage $\mathrm{ER}^{+}$breast cancers. Our findings may be contrasted with the survival study

359 results reported by Hennigs et al. [41] from primary non-metastatic invasive breast cancer

360 patients of different molecular subtypes, but we utilized survival data derived from a

361 longer follow-up with additional $B R C A 1$ gene expression information. Our study still has

362 some limitations. We had expected to acquire a sufficiently large sample size of study

363 subjects from the public domain; however, constrained by different study designs, varied

364 or unclear clinical information items, and inconsistent follow-up periods found in the

365 GSE datasets, it was necessary to adopt the pooled-data approach instead of

366 meta-analysis in this study. Furthermore, the decile value cut at $90 \%$ indicated the best

367 result, but the sample size of this high-BRCAl expression group was small. These factors

368 might alter the statistical results, but the DMFS analysis illustrated by various cut-off

369 points for the $B R C A 1$ expression groups provided with coherent results showing a clear

370 trend. 
371 The high BRCA1 expression patients in this study displayed a biphasic survival curve,

372 which implied that these patients could be divided into two subgroups. The subgroup

373 susceptible to distant metastasis is likely to lose the BRCA1 protein function so that the

374 disseminated cells proliferated fast. In contrast, the non-susceptible subgroup may retain

375 a normal BRCA1 protein level. As a tumor suppressor, normal BRCA1 protein function

376 would lead to DMFS. In other words, these two types of high BRCA1 expression patients

377 may have different outcomes.

378 It is also noted that about $60 \%$ of patients in the pooled cohort were "cured" regardless

379 of the presence of high or low expression of the BRCA1 gene. This observation indicated

380 that BRCAl expression status did not change the lifetime risk of DMFS. This cure

381 proportion may be attributable to the effect of breast surgery combined with adjuvant

382 tamoxifen therapy. Nevertheless, the time to distant metastasis was BRCA1 expression

383 dependent. When the cutoffs were raised from $50 \%$ to $90 \%$, the median onset times of

384 distant metastasis for the susceptible patients of high-BRCAl expression groups were

385 gradually shortened, while those of lower-expression groups consistently retained (Figure 
$3862 \mathrm{~A}$ to $2 \mathrm{D}$ and Figure 3). This observation implied that the onset time might be affected by

387 the proliferation rate of the disseminated tumors. Presumably, the higher the proliferation

388 rate, the earlier the onset time is.

389 In fact, $20 \%$ of sporadic breast tumors [14] did not show BRCA1 staining in either the

390 nucleus or cytoplasm, which demonstrated a defect in protein production. In some cases,

391 breast tumor cells showed cytoplasmic BRCA1 retention along with a loss of nuclear

392 staining [15], which implied a defect in nuclear transportation [42,43]. These BRCA1

393 protein-loss tumor cells were associated with poor prognosis and might induce high

394 BRCAl gene expression via auto-regulation.

395 Some tamoxifen resistant tumors retain responsiveness to estrogen and can be treated by

396 other types of anti-estrogens, such as aromatase inhibitors (AIs) [44]. However, AIs have

397 limitations because they can be only used for post-menopausal patients; therefore, for

398 recurrent tumors with BRCA1 function loss, poly (ADP-ribose) polymerase (PARP)

399 inhibitors might be a good alternative therapy. The PARP inhibitor was developed for

400 BRCA-mutated [45] tumors, but now researchers seek to expand its use to high-risk 
401 patients with similar molecular characteristics [46-48]. BRCA1 protein is involved in the

402 homologous recombination repair pathway to repair DNA double strand breaks. With

403 BRCA1 defects, tumor cells are forced to use alternative DNA-repair pathways [49], such

404 as simple end joining, to fix DNA damage. By inhibiting alternative repair pathways,

405 PARP inhibitors lead tumor cells to die, because all DNA double strand break repair

406 capabilities for tumor cells were blocked. However, normal cells still retain a functional

407 homologous recombination repair pathway, so they can survive quite well upon receiving

408 PARP inhibitor treatment.

409 To develop a companion diagnostic for prescribing PARP inhibitors, the commonly used

410 immunohistochemistry (IHC) staining may not be the best choice since the IHC is

411 cumbersome and is not easy to standardize and automate. Furthermore, the specificity

412 and sensitivity of some IHC antibodies are questionable [50]. In contrast, gene

413 expression-based methods, such as polymerase chain reaction, are specific, sensitive, and

414 quantitative. The latter has the potential to identify which recurrent patients are

415 appropriate for PARP inhibitor therapy. 
416 In summary, our study results can explain the conflicting results produced by previous

417 studies. The high BRCAl gene expression may either favor or not favor the patients'

418 survival. If the high $B R C A 1$ gene expression leads to a high expression of normal

419 BRCA1 protein, the patients appear to be cured. If, however, the high BRCAl gene

420 expression is caused by the loss of BRCA1 protein function, the patients are susceptible

421 to fast distant metastasis.

\section{References}

423 1. Bray F, Ferlay J, Soerjomataram I, Siegel RL, Torre LA, Jemal A. Global cancer

424 statistics 2018: GLOBOCAN estimates of incidence and mortality worldwide for 36

425 cancers in 185 countries. CA Cancer J Clin. 2018;68(6):394-424.

426 2. American Cancer Society. Breast Cancer Facts \& Figures 2019-2020. Am Cancer Soc.

4272019.

428 3. Prat A, Perou CM. Deconstructing the molecular portraits of breast cancer. Mol.

$429 \quad$ Oncol. 2011;5(1):5-23.

4304 Jones LP, Tilli MT, Assefnia S, Torre K, Halama ED, Parrish A, et al. Activation of 
431 estrogen signaling pathways collaborates with loss of Brca1 to promote development

432 of ER $\alpha$-negative and ER $\alpha$-positive mammary preneoplasia and cancer. Oncogene.

$433 \quad 2008 ; 27(6), 794-802$.

434 5. Wang L, Di LJ. BRCA1 and estrogen/estrogen receptor in breast cancer: Where they

435 interact? Int. J. Biol. Sci. 2014; 10(5):566-75.

436 6. Ciocca DR, Fanelli MA. Estrogen receptors and cell proliferation in breast cancer.

437 Trends Endocrinol. Metab. 1997;8(8):313-21.

438 7. Liao XH, Lu DL, Wang N, Liu LY, Wang Y, Li YQ, et al. Estrogen receptor $\alpha$

439 mediates proliferation of breast cancer MCF-7 cells via a p21/PCNA/E2F1-dependent

440 pathway. FEBS J. 2014;281(3):927-42.

441 8. Tian JM, Ran B, Zhang CL, Yan DM, Li XH. Estrogen and progesterone promote

442 breast cancer cell proliferation by inducing cyclin G1 expression. Brazilian J Med

$443 \quad$ Biol Res. 2018;51(3):e5612.

444 9. Pan H, Gray R, Braybrooke J, Davies C, Taylor C, McGale P, et al. 20-Year Risks of

445 Breast-Cancer Recurrence after Stopping Endocrine Therapy at 5 Years. N Engl J 
$446 \quad$ Med. 2017;377(19):1836-46.

447 10. Wen J, Li R, Lu Y, Shupnik MA. Decreased BRCA1 confers tamoxifen resistance in

448 breast cancer cells by altering estrogen receptor-coregulator interactions. Oncogene.

$449 \quad 2009 ; 28(4): 575-86$.

450 11. Wu J, Lu LY, Yu X. The role of BRCA1 in DNA damage response. Protein Cell.

$451 \quad 2010 ; 1(2): 117-23$.

452 12. Davies M. BRCA1 and estrogen receptor signaling. Breast Cancer Res.

$453 \quad$ 1999;1:66580.

454 13. Fan S, Wang JA, Yuan R, Ma Y, Meng Q, Erdos MR, et al. BRCA1 inhibition of

455 estrogen receptor signaling in transfected cells. Science. 1999;284:1354-6.

456 14. Taylor J, Tymboura M, Pace PE, A'Hern RP, Desai AJ, Shousha S, et al. An

457 important role for BRCA1 in breast cancer progression is indicated by its loss in a

458 large proportion of non-familial breast cancers. Int J Cancer. 1998;79(4):334-42.

459 15. Mahmoud AM, Al-Alem U, Macias V, Deaton RJ, Balla A, Gann P, et al. BRCA1

460 protein expression and subcellular localization in primary breast cancer: Automated 
461 digital microscopy analysis of tissue microarrays. PLoS One. 2017;12(9):e0184385.

462 16. Hedau S, Batra M, Singh UR, Bharti AC, Ray A, Das BC. Expression of BRCA1 and

463 BRCA2 proteins and their correlation with clinical staging in breast cancer. J Cancer

$464 \quad$ Res Ther. 2015;11(1):158-63.

465 17. Tung N, Miron A, Schnitt SJ, Gautam S, Fetten K, Kaplan J, et al. Prevalence and

466 predictors of loss of wild type BRCA1 in estrogen receptor positive and negative

467 BRCA1-associated breast cancers. Breast Cancer Res. 2010;12(6):R95.

468 18. Yoshikawa K, Honda K, Inamoto T, Shinohara H, Yamauchi A, Suga K, et al.

469 Reduction of BRCA1 protein expression in Japanese sporadic breast carcinomas and

470 its frequent loss in BRCA1-associated cases. Clin Cancer Res. 1999;5(6):1249-61.

471 19. Fraser JA, Reeves JR, Stanton PD, Black DM, Going JJ, Cooke TG, et al. A role for

472 BRCA1 in sporadic breast cancer. Br J Cancer. 2003;88(8):1263-70.

473 20. De Siervi A, De Luca P, Byun JS, Di LJ, Fufa T, Haggerty CM, et al. Transcriptional

474 autoregulation by BRCA1. Cancer Res. 2010;70(2):532-42.

475 21. Zghair AN, Sharma R, Alfaham M, Sharma AK. Upregulation of BRCA1, ERBB2 
and TP53 marker genes expression in breast cancer patients. Int J Pharm Res. 2018;

477 10(2):147-54.

478 22. Gudas JM, Li T, Nguyen H, Jensen D, Rauscher FJ, Cowan KH. Cell cycle regulation 479 of BRCA1 messenger RNA in human breast epithelial cells. Cell Growth Differ.

$480 \quad 1996 ; 7(6): 717-23$.

481 23. Favy DA, Lafarge S, Rio P, Vissac C, Bignon YJ, Bernard-Gallon D. Real-time PCR

482 quantification of full-length and exon 11 spliced BRCA1 transcripts in human breast

$483 \quad$ cancer cell lines. Biochem Biophys Res Commun. 2000;274(1):73-8.

484 24. Zhu Y, Liu Y, Zhang C, Chu J, Wu Y, Li Y, et al. Tamoxifen-resistant breast cancer

485 cells are resistant to DNA-damaging chemotherapy because of upregulated BARD1

$486 \quad$ and BRCA1. Nat Commun. 2018;9(1):1595.

487 25. Thompson ME, Jensen RA, Obermiller PS, Page DL, Holt JT. Decreased expression

488 of BRCA1 accelerates growth and is often present during sporadic breast cancer

489 progression. Nat Genet. 1995;9:444-50.

490 26. Rice JC, Massey-Brown KS, Futscher BW. Aberrant methylation of the BRCA1 CpG 
491 island promoter is associated with decreased BRCA1 mRNA in sporadic breast cancer cells. Oncogene. 1998;17:1807-12.

493 27. Sourvinos G, Spandidos DA. Decreased BRCA1 expression levels may arrest the cell 494 cycle through activation of p53 checkpoint in human sporadic breast tumors.

495 Biochem Biophys Res Commun. 1998;245:75-80.

496 28. Seery LT, Knowlden JM, Gee JM, Robertson JF, Kenny FS, Ellis IO, et al. BRCA1

497 expression levels predict distant metastasis of sporadic breast cancers. Int J Cancer.

$498 \quad 1999 ; 84: 258-62$.

499 29. Chen CH, Tsay YC, Wu YC, Horng CF. Logistic-AFT location-scale mixture

500 regression models with nonsusceptibility for left-truncated and general

501 interval-censored data. Stat Med. 2013;32(24):4285-305.

502 30. Cox DR. Regression Models and Life-Tables. J R Stat Soc Ser B.

$503 \quad 1972 ; 34(2): 187-220$.

504 31. Home-GEO-NCBI. Gene Expression Omnibus [https://www.ncbi.nlm.nih.gov/geo/].

505 Accessed: 25 Sept. 2020 
506 32. Gruosso T, Mieulet V, Cardon M, Bourachot B, Kieffer Y, Devun F, et al. Chronic

507 oxidative stress promotes $\mathrm{H} 2 \mathrm{AX}$ protein degradation and enhances chemosensitivity

508 in breast cancer patients. EMBO Mol Med. 2016;8:527-49.

509 33. Zhang Y, Sieuwerts AM, McGreevy M, Casey G, Cufer T, Paradiso A, et al. The

510 76-gene signature defines high-risk patients that benefit from adjuvant tamoxifen

511 therapy. Breast Cancer Res Treat. 2009;116(2):303-9.

512 34. Symmans WF, Hatzis C, Sotiriou C, Andre F, Peintinger F, Regitnig P, et al. Genomic

513 index of sensitivity to endocrine therapy for breast cancer. J Clin Oncol.

$514 \quad 2010 ; 28(27): 4111-9$.

515 35. Nagalla S, Chou JW, Willingham MC, Ruiz J, Vaughn JP, Dubey P, et al. Interactions

516 between immunity, proliferation and molecular subtype in breast cancer prognosis.

$517 \quad$ Genome Biol. 2013;14(4):R34

518 36. Gautier L, Cope L, Bolstad BM, Irizarry RA. Affy - Analysis of Affymetrix

519 GeneChip data at the probe level. Bioinformatics. 2004;20:307-15.

520 37. Choe SE, Boutros M, Michelson AM, Church GM, Halfon MS. Preferred analysis 
521 methods for Affymetrix GeneChips revealed by a wholly defined control dataset.

$522 \quad$ Genome Biol. 2005;6(2):R16.

523 38. Li Q, Birkbak NJ, Gyorffy B, Szallasi Z, Eklund AC. Jetset: Selecting the optimal

524 microarray probe set to represent a gene. BMC Bioinformatics. 2011;12:474.

525 39. Kaplan EL, Meier P. Nonparametric Estimation from Incomplete Observations. J Am

$526 \quad$ Stat Assoc. 1958;53(282):457-81.

527 40. Tsay YC, Chen CH, Wu YC, Horng CF, et al. CRAN-Package MixtureREgLTIC.

528 MixtureRegLTIC: Mixture Regression Models for Left-Truncated and

529 Interval-Censored Data.

530 [https://cran.r-project.org/web/packages/MixtureRegLTIC/index.html]. Accessed: 25

$531 \quad$ Sept. 2020

532 41. Hennigs A, Riedel F, Gondos A, Sinn P, Schirmacher P, Marmé F, et al. Prognosis of

533 breast cancer molecular subtypes in routine clinical care: A large prospective cohort

$534 \quad$ study. BMC Cancer. 2016;16(1):734.

535 42. Yang ES, Xia F. BRCA1 16 years later: DNA damage-induced BRCA1 shuttling. 
FEBS J. 2010;277(15):3079-85.

537 43. Alshareeda AT, Negm OH, Green AR, Nolan CC, Tighe P, Albarakati N, et al.

538 KPNA2 is a nuclear export protein that contributes to aberrant localisation of key

539 proteins and poor prognosis of breast cancer. Br J Cancer. 2015;112(12):1929-37.

540 44. Fabian CJ. The what, why and how of aromatase inhibitors: hormonal agents for

541 treatment and prevention of breast cancer. Int J Clin Pract. 2007:61(12):2051-63.

542 45. Byrum AK, Vindigni A, Mosammaparast N. Defining and Modulating 'BRCAness.'

543 Trends in Cell Biology. 2019:29(9):740-51.

544 46. Shao N, Shi Y, Yu L, Ye R, Shan Z, Zhang Z, et al. Prospect for Application of PARP

545 Inhibitor in Patients with HER2 Negative Breast Cancer. Int. J. Biol. Sci.

$546 \quad 2019 ; 15(5): 962-72$.

547 47. Pulliam N, Fang F, Ozes AR, Tang J, Adewuyi A, Keer H, et al. An effective

548 epigenetic-PARP inhibitor combination therapy for breast and ovarian cancers

549 independent of BRCA mutations. Clin Cancer Res. 2018;24(13):3163-75.

550 48. McCann KE, Hurvitz SA. Advances in the use of PARP inhibitor therapy for breast 
$551 \quad$ cancer. Drugs Context. 2018;7:212540.

552 49. Rodgers K, Mcvey M. Error-Prone Repair of DNA Double-Strand Breaks. J. Cell.

$553 \quad$ Physiol. 2016;231(1):15-24.

554 50. Al-Mulla F, Abdulrahman M, Varadharaj G, Akhter N, Anim JT. BRCA1 gene

555 expression in breast cancer: a correlative study between real-time RT-PCR and

556 immunohistochemistry. J Histochem Cytochem. 2005;53(5):621-9.

\section{Declarations}

558 Ethics approval and consent to participate

559 This work is a pooled analysis of public data from gene expression omnibus (GEO) at the

560 National Center for Biotechnology Information. There is no need to get ethics approval.

\section{Consent for publication}

562 All authors agreed for this submission.

\section{Availability of data and material}

564 This work only analyzed public data; no new data were generated.

\section{Competing interests}


566 All authors declared no competing interests.

\section{Funding}

568 This work is partially supported by funding from 3 sources: 1) grant 103-2911-I-010-514

569 from Ministry of Science and Technology, Taiwan; 2) grant from 109-2321-B-002-038

570 from Ministry of Science and Technology, Taiwan; 3) Intramural Research Program of

571 National Institute of Neurological Disorders and Stroke, National Institutes of Health,

572 USA.

573 Authors' contributions

574 Hui-Ju Chang: conceived the idea, carried out the analysis, wrote the manuscript

575 Ueng-Cheng Yang: conceived the idea, revised the manuscript

576 Mei-Yu Lai: performed the cure model analysis

577 Chen-Hsin Chen: developed the logistic AFT cure model, revised the manuscript

578 Yang-Cheng Fann: helped with the programming, revised manuscript

\section{Acknowledgements}

580 We thank Mr. Wei-Hung Chuang for helpful discussion. 
Subtypes

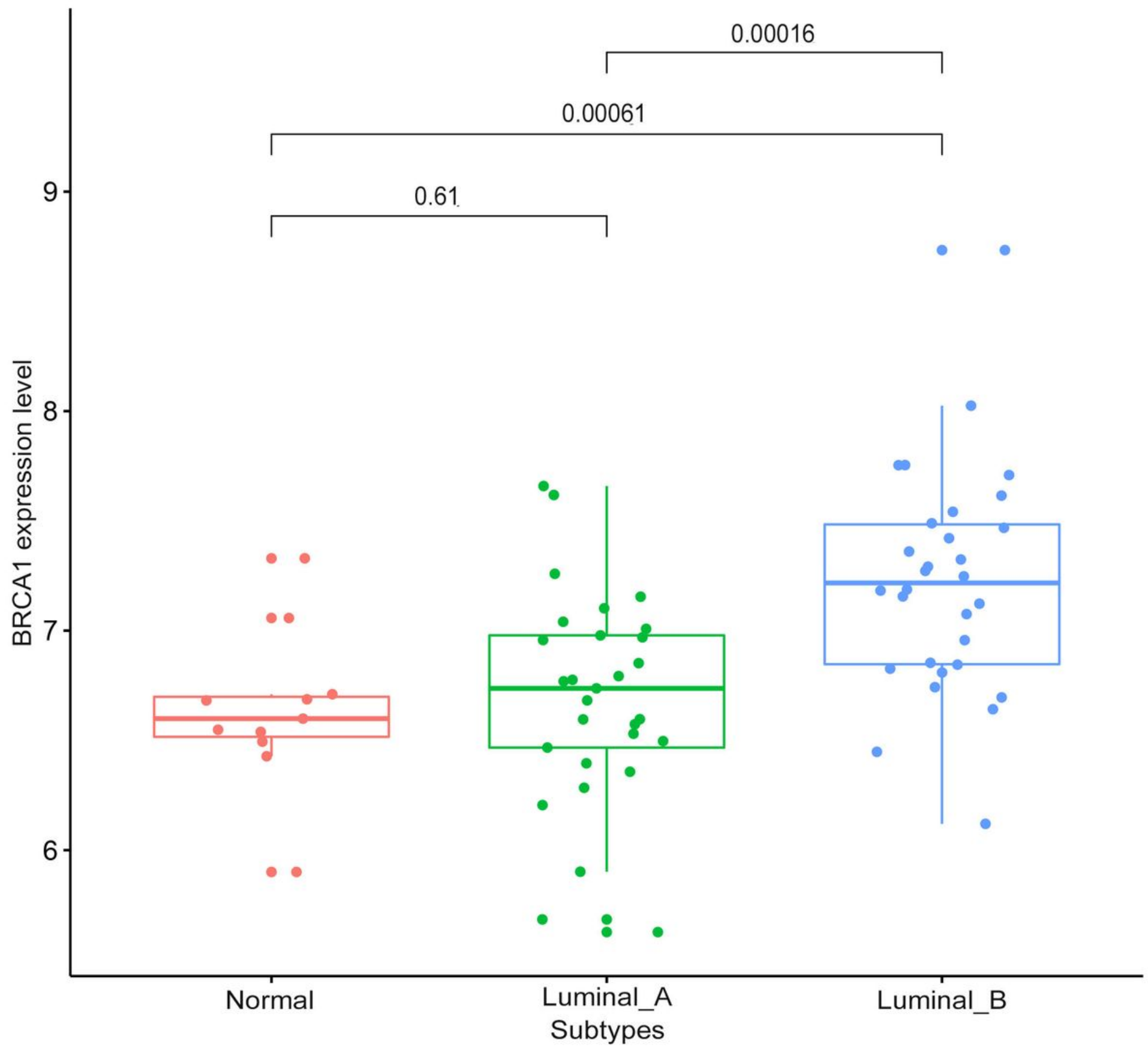

\section{Figure 1}

Boxplots of BRCA1 expression in normal breast tissue, Luminal A and Luminal B tumors of GSE45827. Normal breast tissues (red), luminal A tumors (green) and luminal B tumors (blue) are displayed from left to right. Each dot represents the BRCA1 expression level of a certain sample. Pairwise $p$-values between different subtypes were calculated using the Kruskal-Wallis test. 

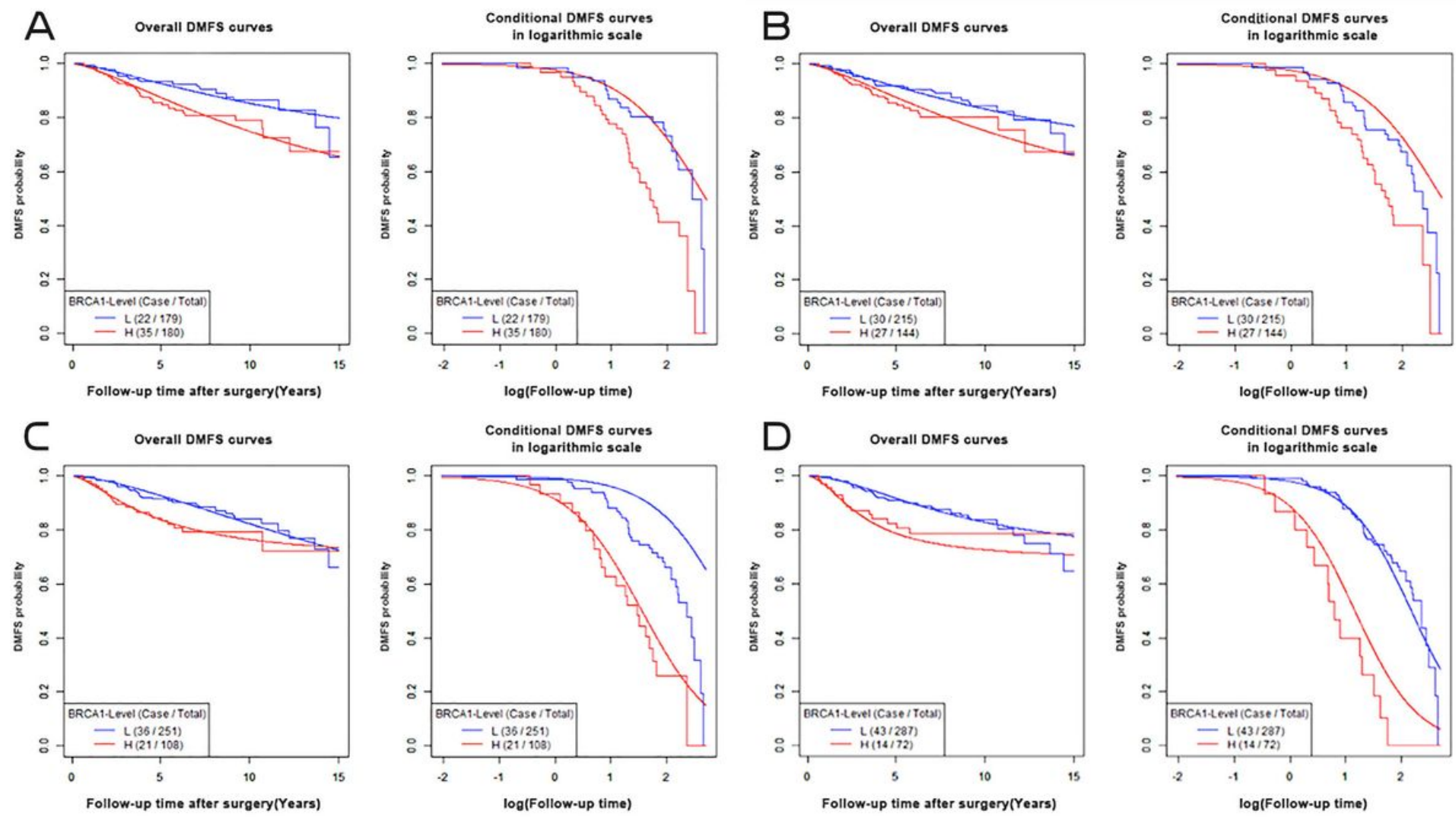

\section{Figure 2}

Survival curves fitted by the "Logistic-AFT mixture regression model" at the 5th-8th deciles. Kaplan-Meier (step function) and the mixture regression (smooth curve) estimators of overall and conditional DMFS curves for the distant metastasis onset time after the surgery stratified by BRCA1-level defined at the cutoff point (A) 50th, (B) 60th, (C) 70th, and (D) 80th percentile, and corresponding $p$ values of likelihood ratio test (PLRT) were $0.011,0.038,0.014$ and 0.006 , respectively. The BRCA1 high- and low-expression groups were shown in red and in blue, respectively, for each cut-off point. 

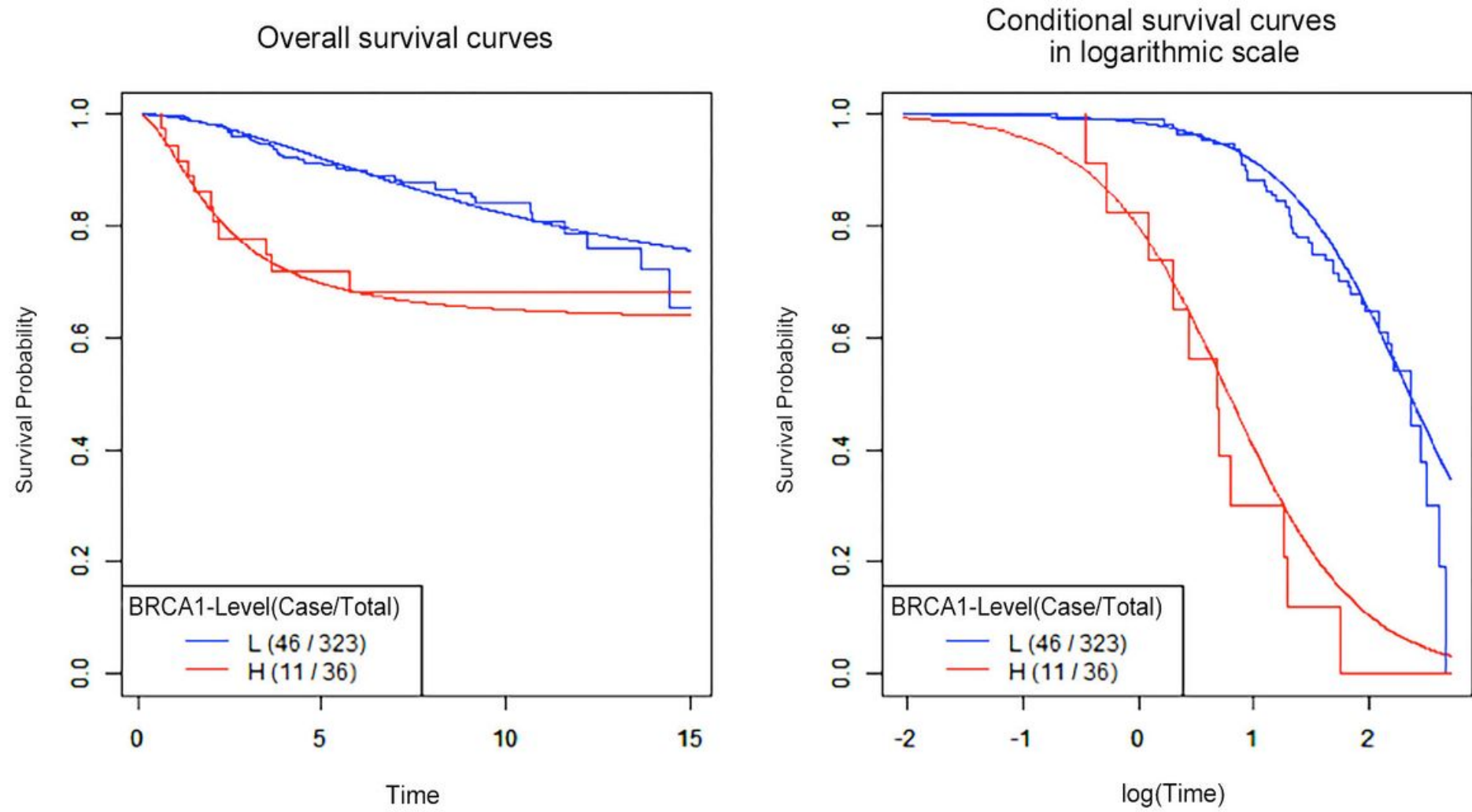

Figure 3

Survival curves fitted by the "Logistic-AFT mixture regression model" at the 9th decile. Kaplan-Meier (step function) and the mixture regression (smooth curve) estimators of overall and conditional DMFS curves for the distant metastasis onset time after the surgery stratified by BRCA1-level defined at the cut-off point 90th percentile. The BRCA1 high- and low-expression groups were shown in red and in blue, respectively.

\section{Supplementary Files}

This is a list of supplementary files associated with this preprint. Click to download.

- SupplementaryTableS1.docx

- SupplementaryTableS2.docx 